\title{
Intra-articular venous malformation of the knee in children: magnetic resonance imaging findings and significance of synovial involvement
}

\author{
Katariina A. Mattila ${ }^{1}$ (D) $\cdot$ Johanna Aronniemi ${ }^{2,3} \cdot$ Päivi Salminen ${ }^{1,3} \cdot$ Risto J. Rintala $^{1} \cdot$ Kristiina Kyrklund $^{1,3}$
}

Received: 29 May 2019 / Revised: 2 September 2019 / Accepted: 14 November 2019/Published online: 19 December 2019

(C) The Author(s) 2019

\begin{abstract}
Background Intra-articular venous malformations of the knee are an uncommon cause of unilateral knee pain in children. Timely diagnosis is important because lesions with intrasynovial involvement can lead to joint space hemorrhage and secondary cartilage damage.

Objective To describe our tertiary center's experience of diagnostics and typical magnetic resonance imaging (MRI) findings. Materials and methods A retrospective review of all patients $\leq 16$ years of age managed for intra-articular venous malformations of the knee at our institution between 2002 and 2018.

Results Of 14 patients ( 8 male), the mean age at presentation was 6 years (range: $0-14$ years). The most common clinical findings were unilateral knee pain (93\%), joint swelling (79\%), quadriceps atrophy (50\%) and a limited range of motion (29\%). Cutaneous manifestations were present in four patients (29\%). Contrast-enhanced MRI was available in all cases. After initial MRI, a vascular anomaly etiology had been identified in 11 cases (79\%), and correctly reported as a venous malformation in $6(55 \%)$. Three patients received entirely different diagnoses (arthritis, tumor or pigmented villonodular synovitis). Three of seven patients with intrasynovial lesions had established chondropathy at diagnosis. Two patients with lesions of the suprapatellar fat pad had intrasynovial involvement that was not visualised on MRI.

Conclusion Although MRI usually permits the diagnosis, clinical awareness of these lesions is important for optimal imaging, accurate interpretation and timely diagnosis. Involvement of the intrasynovial cavity carries a risk of hemarthrosis and progressive chondropathy that may be underestimated by MRI.
\end{abstract}

Keywords Children $\cdot$ Intra-articular $\cdot$ Knee $\cdot$ Magnetic resonance imaging $\cdot$ Synovium $\cdot$ Venous malformation

\section{Introduction}

Venous malformations are benign congenital, slow-flow vascular anomalies consisting of thin-walled and dilated venous channels $[1,2]$. Intra-articular venous malformations of the knee are a rare

Katariina A. Mattila

katariina.mattila@helsinki.fi

1 Department of Pediatric Surgery, Children's Hospital, Helsinki University Hospital and University of Helsinki, P.O. Box 347, 00029 HUS, Helsinki, Finland

2 Department of Radiology, HUS Medical Imaging Center, Helsinki University Hospital and University of Helsinki, Helsinki, Finland

3 VASCERN VASCA European Reference Center, Helsinki University Hospital, Helsinki, Finland subtype of venous malformations that are an uncommon cause of knee pain in children. Presenting symptoms often include nonspecific pain and joint swelling, which may be episodic, accompanied by a limited range of motion. Secondary muscle atrophy may be present if symptoms are prolonged. External signs suggestive of a venous malformation may include varicosities or capillary malformations on the skin surface or, more rarely, overgrowth or undergrowth of the affected limb. However, superficial skin changes may also be absent, and the symptoms and signs display overlap with those of other more common conditions including juvenile idiopathic arthritis, trauma or even tumors $[3,4]$. Timely diagnosis of intra-articular venous malformations of the knee is essential because lesions with intrasynovial involvement can lead to joint space hemorrhage and secondary cartilage damage, which is the most significant complication [4-7] (Fig. 1). Hence, improving clinical awareness of venous malformations and familiarity with their magnetic resonance imaging (MRI) characteristics are beneficial. 


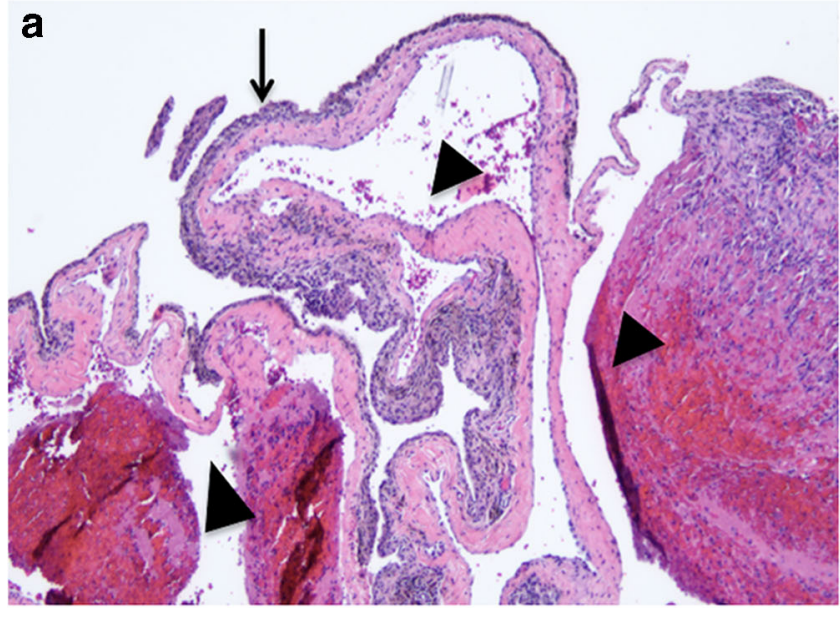

Fig. 1 Paraffin sections of a resected synovial venous malformation demonstrate post-hemorrhage iron accumulation at the synovium. a The synovium is staining dark purple at herovici staining (arrow). Dilated venous spaces with intraluminal thrombi are right beneath the

This study aims to highlight intra-articular venous malformation of the knee as a rare but important differential diagnostic cause of unilateral knee pain in children. We describe our institutional experience in diagnosing these lesions, as well as their typical MRI findings against other diagnoses. We also emphasize the importance of evaluating and recognizing possible synovial involvement.

\section{Materials and methods}

This study was carried out at the Children's Hospital, Helsinki University Hospital, which is a tertiary referral centre for vascular anomalies in children. Helsinki University Hospital founded an interdisciplinary team in 2002 to diagnose and treat vascular anomalies. The team includes diagnostic and interventional radiology, pediatric, plastic, maxillofacial and orthopedic surgery, oncology, otorhinolaryngology, ophthalmology, dermatology, genetics and pathology.

The case records and imaging of all children $\leq 16$ years of age with intra-articular venous malformations of the knee managed at our institution between 2002 and 2018 were retrospectively reviewed. Referral diagnoses were compared with definitive diagnoses established by our vascular anomaly team. Definitive diagnoses were based on the updated International Society for the Study of Vascular Anomalies (ISSVA) classification [8]. MRI studies were analyzed for morphology, enhancement and anatomical locations of the lesions, and to review the need for further imaging for diagnostics. Lesions with entire or partial location within the joint capsule were considered intra-articular. Based on imaging, intra-articular lesions were further subdivided into: 1) intracapsular but extrasynovial, including the suprapatellar and infrapatellar fat pads, or 2) intracapsular and

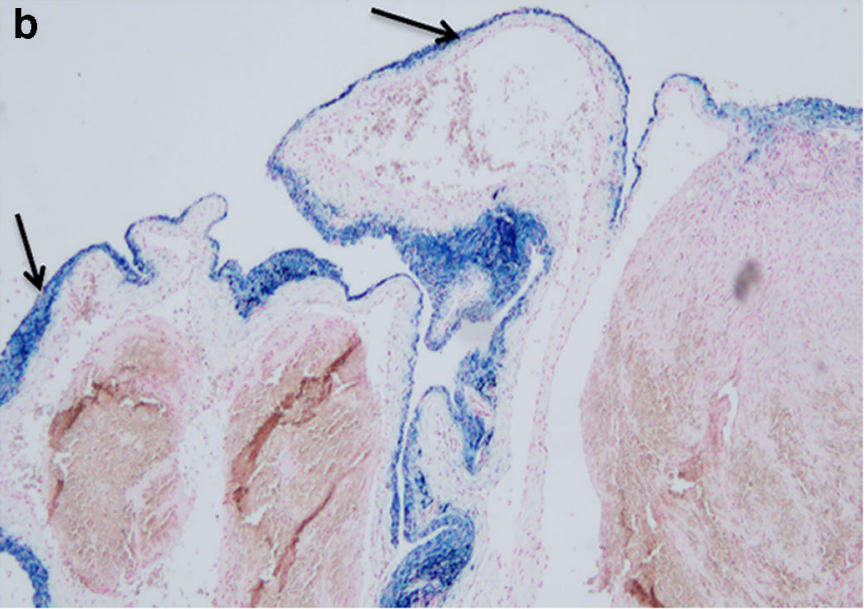

synovium (arrowheads). b At Prussian blue staining, synovial iron stains blue (arrows). Fig. 1a and b are a 40x magnification. Image courtesy of Dr. Jouko Lohi

intrasynovial, when entirely or partially affecting the intrasynovial cavity. Involvement of extracapsular tissues was also recorded.

\section{Results}

\section{Clinical data}

Fourteen pediatric patients ( 8 males) with intra-articular venous malformation of the knee were identified. The mean age at presentation was 6 years (range: $0-14$ years). The most common presenting symptoms and signs were unilateral knee pain (13/14), joint swelling (11/14), quadriceps atrophy (7/14) and a limited range of motion (4/14). Cutaneous manifestations suggestive of a vascular anomaly were present in four patients: superficial varicosities in one, and regional skin surface capillary malformations in three. The Ddimer level, which may be elevated in venous malformations due to localized intravascular coagulopathy [7, 9], was raised ( $>0.5 \mathrm{mg} / \mathrm{l})$ in nine patients (mean: $1.7 \mathrm{mg} / \mathrm{l}$, range: $0.0-5.5$ ).

\section{Referral diagnoses and initial magnetic resonance imaging diagnoses}

The mean time from the onset of symptoms to the definitive diagnosis was 2 years (range: 0-9 years). Patient characteristics, referral diagnoses and initial diagnoses following MRI are presented in Table 1. After initial MRI, a vascular anomaly etiology was identified in 11 patients (79\%), and correctly reported as a venous malformation in 6 patients (55\%), an unspecified vascular anomaly in 2 patients $(18 \%)$ and incorrectly as a hemangioma in 3 patients $(27 \%)$. Three patients received entirely other diagnoses based on initial clinical assessment and imaging (arthritis, tumor or pigmented 
Table 1 Patient characteristics and time to diagnosis

\begin{tabular}{llllll}
\hline Patient & $\begin{array}{l}\text { Age at first } \\
\text { contact }\end{array}$ & Time to diagnosis & Referral diagnosis & $\begin{array}{l}\text { First magnetic resonance } \\
\text { imaging report }\end{array}$ & $\begin{array}{l}\text { Diagnosis after assessment by } \\
\text { vascular anomalies team and/or } \\
\text { further imaging }\end{array}$ \\
\hline 1 & 2 years & 5 months & arthritis & venous malformation & venous malformation \\
2 & 7 years & 2 months & reactive arthritis & hemangioma & venous malformation \\
3 & 2 years & 2 years, 4 months & juvenile idiopathic arthritis & pigmented villonodular synovitis & venous malformation \\
4 & 4 years & 4 years, 7 months & juvenile idiopathic arthritis & arthritis & venous malformation \\
5 & 8 years & 3 years, 10 months & juvenile idiopathic arthritis & venous malformation & venous malformation \\
6 & 10 years & 0 months & hemangioma & hemangioma & venous malformation \\
7 & 7 years & 9 years, 1 month & hemangioma & hemangioma & tumour \\
8 & 4 years & 2 months & tumour & unspecified vascular anomaly & venous malformation \\
9 & 0 years & 0 months & tumour & unspecified vascular anomaly-venous malformation \\
10 & 3 years & 0 months & unspecified vascular anomaly & venous malformation \\
11 & 6 years & 2 years, 8 months & venous malformation & venous malformation & venous malformation \\
12 & 14 years & 0 months & venous malformation & venous malformation & venous malformation \\
13 & 12 years & 0 months & venous malformation & venous malformation & venous malformation \\
14 & 11 years & 0 months & capillary-venous malformation & venous malformation & capillary-venous malformation
\end{tabular}

villonodular synovitis) and were referred after a delay following tissue biopsy or assessment of joint aspirate. The MRI protocol varied depending on the imaging practices of the referral units. In cases with insufficient sequences for tissue characterization (T1- and T2-weighted turbo spin echo and contrast-enhanced sequences), the study was subsequently performed at our institution.

\section{Imaging findings}

Contrast-enhanced MRI (1.5 T) of the knee was assessed in all patients. The venous malformations appeared as collections of tubular structures with high signal intensity on T2-weighted sequences (Fig. 2). The lesion morphology was well demarcated and cluster-like in half of the patients (Figs. 2 and 3), the other half being diffuse and phlebectatic (Figs. 4 and 5). The enhancement pattern after gadolinium was partial and progressively increased in consecutive sequences in 10 patients (Fig. 3). However, in diffuse lesions with narrower vascular spaces, the enhancement was intense beginning from the first post-contrast images (Fig. 5). Phleboliths or thrombosis was present in $79 \%$ of the lesions (Figs. 3 and 6).

The suprapatellar fat pad was the most common location affected (12/14 patients) (86\%) (Fig. 2). Overall, the venous malformation appeared intracapsular but extrasynovial in 7 patients $(50 \%)$ and intracapsular with intrasynovial extension in the remaining 7 (Figs. 2, 3, 4 and 5). A synovial effusion was present in 4 patients (29\%), all with intrasynovial
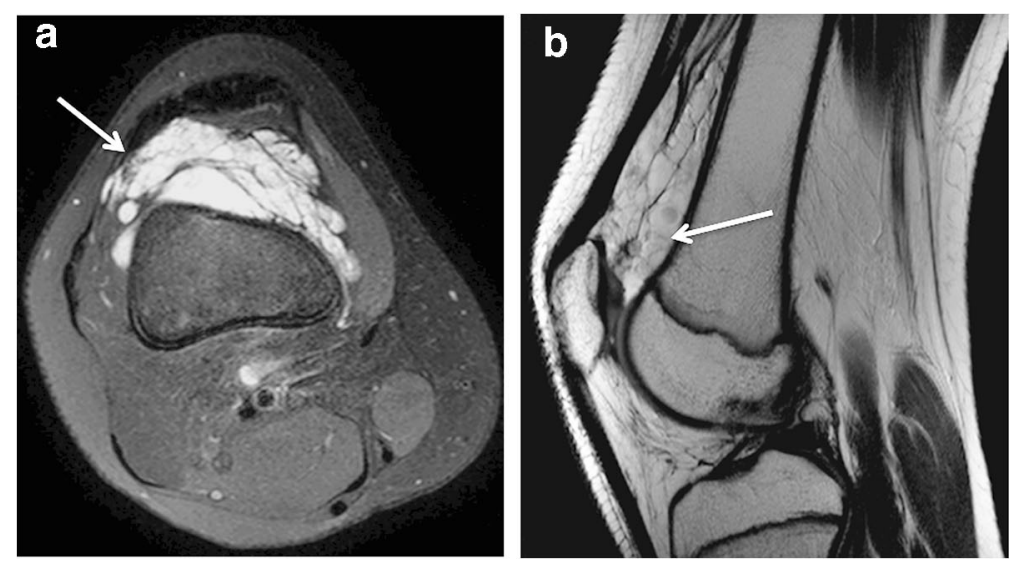

Fig. 2 A 12-year-old girl with a venous malformation of the suprapatellar fat pad. a A T2-weighted fat-saturated axial MRI shows a typical clusterlike lesion consisting of tubular structures (arrow). b A T2-weighted sagittal MRI demonstrates the close proximity of the suprapatellar synovial recess and the venous malformation. The outline of the synovium is not identifiable between the venous malformation and femoral cortex (arrow) and possible synovial involvement cannot be ruled out. c A T1-weighted fat-saturated MRI shows partial enhancement of the lesion after gadolinium injection (arrow) 
Fig. 3 A 7-year-old boy with an intraarticular and intrasynovial venous malformation of the knee. a, b Axial (a) and sagittal (b) T2weighted fat-saturated MR images. The cluster-like venous malformation protrudes into the suprapatellar recess (arrow in a). A mild synovial effusion with post-hemorrhage debris is present (arrowhead in a). The venous malformation invades both the suprapatellar and infrapatellar fat pads (arrows in b) as well as the intrasynovial cavity (arrowhead in b). c, d T1-weighted fatsaturated post-contrast axial (c) and coronal (d) MR images demonstrate the progressive enhancement typical for venous malformations. The first postcontrast image (c) shows only mild and partial enhancement of the venous malformation (arrow). A consecutively obtained coronal image (d) demonstrates more extensive enhancement. The filling defects (arrow in d) are due to intraluminal thrombosis often present in venous malformations
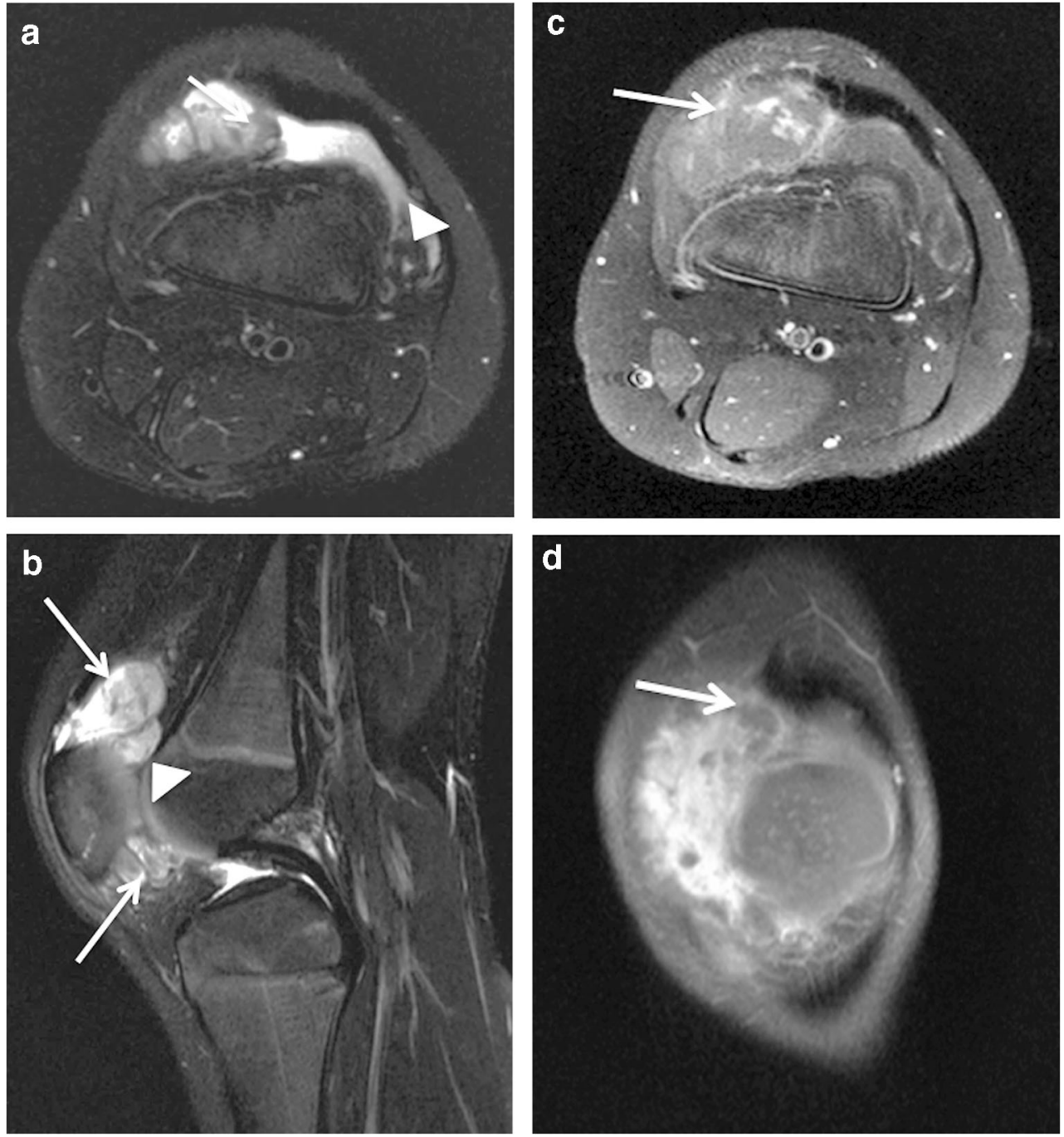

involvement (Fig. 3). Three of the 7 clearly intrasynovial lesions $(43 \%)$ were associated with established chondral damage, characterized by thinning of the articular surface cartilage, full thickness cartilage defects and osteochondral lesions (Fig. 4). Extracapsular tissues (muscle, subcutaneous fat, bone) were affected in 10 of the patients (71\%; Figs. 5 and 6). Among the 12 patients with suprapatellar fat pad lesions, 2 lesions that appeared to have only extrasynovial components on MRI were found to have intrasynovial extension during subsequent operative management. In these cases, no synovial effusion was present at the time of MRI (Fig. 2).

\section{Discussion}

Intra-articular venous malformation of the knee is a rare but important entity in children. Consistent with the literature and our series, knee pain and swelling due to intra-articular venous malformations may be mistaken for juvenile idiopathic arthritis $[3,4]$, pigmented villonodular synovitis or tumors.
Cutaneous changes, including superficial venous or capillary malformations, were important clinical clues that led to identifying the venous malformation or at least suspicion of a vascular anomaly as the etiology; however, external signs were only present in $29 \%$ of cases. In pediatric patients, persistent unilateral knee pain is an abnormal symptom in any case that warrants further investigation. Clinical awareness of intra-articular venous malformations among the spectrum of unusual diagnoses is perhaps most important in prompting further imaging. MRI is pivotal in the diagnostics of intraarticular venous malformation of the knee [10] and in evaluating their anatomical location and possible sequelae. In the context of venous malformations of the limb, clinical risk factors for subsequent joint dysfunction were an age younger than 10 years, and pain duration over 1 year according to a recent series [11].

The imaging work-up of nonspecific knee pain in pediatric patients should always include a plain film x-ray, which is often normal in intra-articular venous malformations. Phleboliths, when present, may be visible on x-ray and raise 


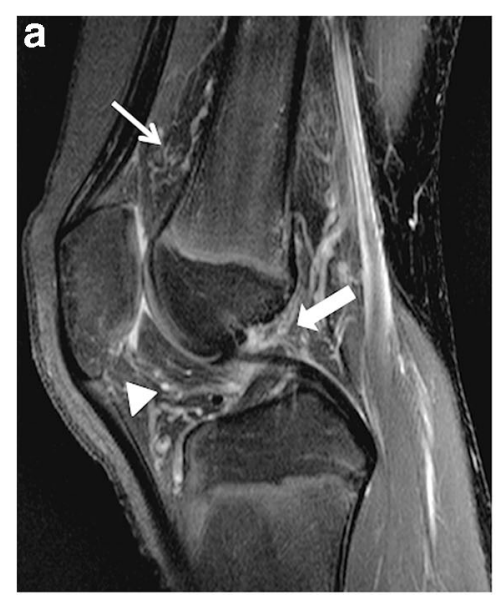

Fig. 4 A 13-year-old boy with a diffuse intra-articular venous malformation of the knee. a A T2-weighted fat-saturated sagittal MRI shows the diffusely infiltrating phlebectatic vessels affecting the suprapatellar fat pad (thin arrow), infrapatellar fat pad (arrowhead) and intrasynovial cavity at the level of intercondylar space (thick arrow). b An axial T2-weighted fat-saturated MRI at the level of the intercondylar space demonstrates the pathological veins invading the intercondylar space and thus intrasynovial cavity (arrow). c A T2-weighted fatsaturated coronal MRI demonstrates thinning of cartilages and a fullthickness cartilage defect (arrow) of the same knee developed as a result of recurrent intrasynovial hemorrhages

varies from lobulated cluster-like structures (Fig. 2) to diffusely infiltrating phlebectasia (Figs. 4 and 5) [12, 13]. Phleboliths, the small intravenous calcifications, appear as small signal void foci in both $\mathrm{T} 1$ - and $\mathrm{T} 2$-weighted sequences (Fig. 6). They are hallmarks for venous malformations but are not uniformly present [14]. T2 turbo spin echo sequence in the sagittal plane is particularly useful in evaluating the exact anatomy of the malformation and possible synovial involvement in the intercondylar space or suprapatellar recess (Fig. 2). Intravenous gadolinium helps characterize tissue and confirm the vascular nature of the lesion; as venous malformations are connected to the systemic circulation via capillaries, they fill with the contrast agent. The filling rate is variable depending on the flow velocity and the size of the
Fig. 5 An 11-year-old boy with an extensive and diffuse venous malformation of the lower extremity. a A T2-weighted fatsaturated sagittal MRI demonstrates the involvement of all intracapsular spaces including the synovial cavity in the intercondylar space (arrow), as well as extracapsular muscle involvement (arrowheads). b A T1-weighted fat-saturated postcontrast axial MRI at the level of the intercondylar space demonstrates intensive enhancement of the ectatic veins of the malformation (arrow)
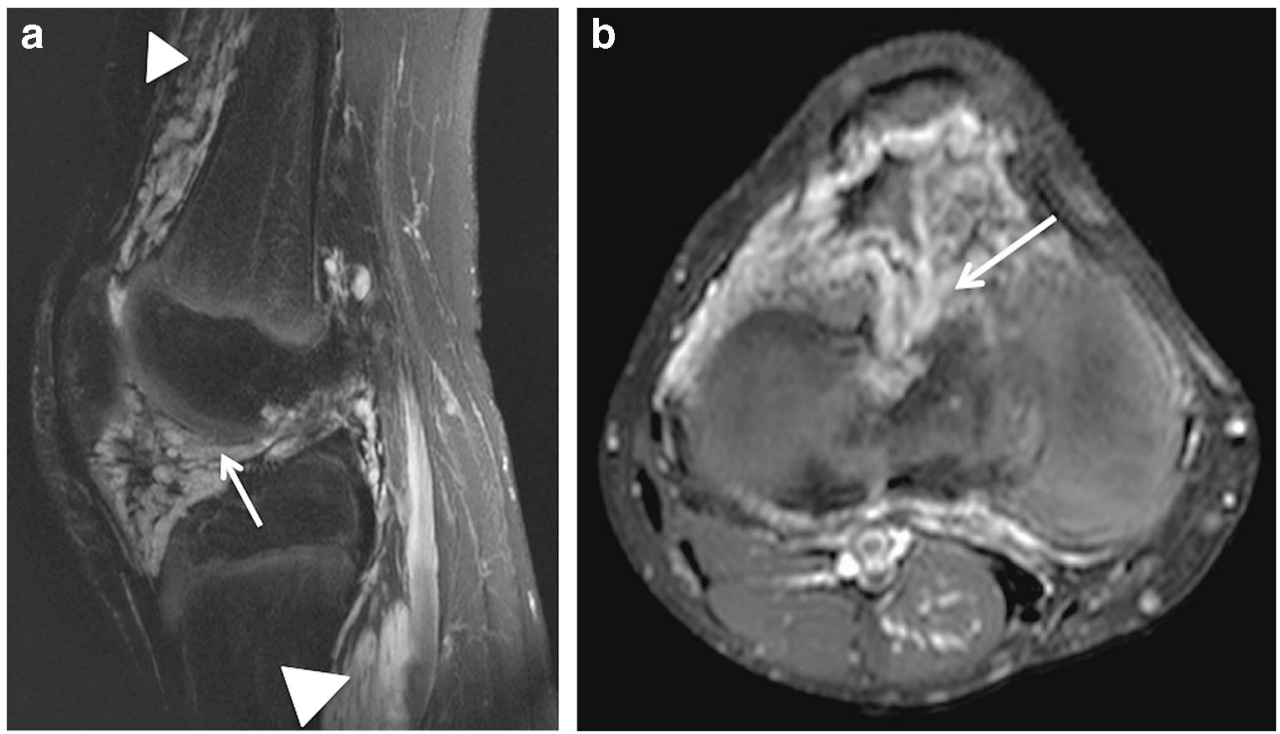
Fig. 6 A 13-year-old girl with a venous malformation of the knee that affects both intra- and extracapsular tissues. a, b T2weighted fat-saturated axial (a) and coronal (b) magnetic resonance images demonstrate the venous malformation invading the distal end of the vastus medialis muscle (thin arrows) and the suprapatellar fat pad (arrowhead). The small lowsignal foci are consistent with intravascular thrombi or phleboliths (thick arrows)
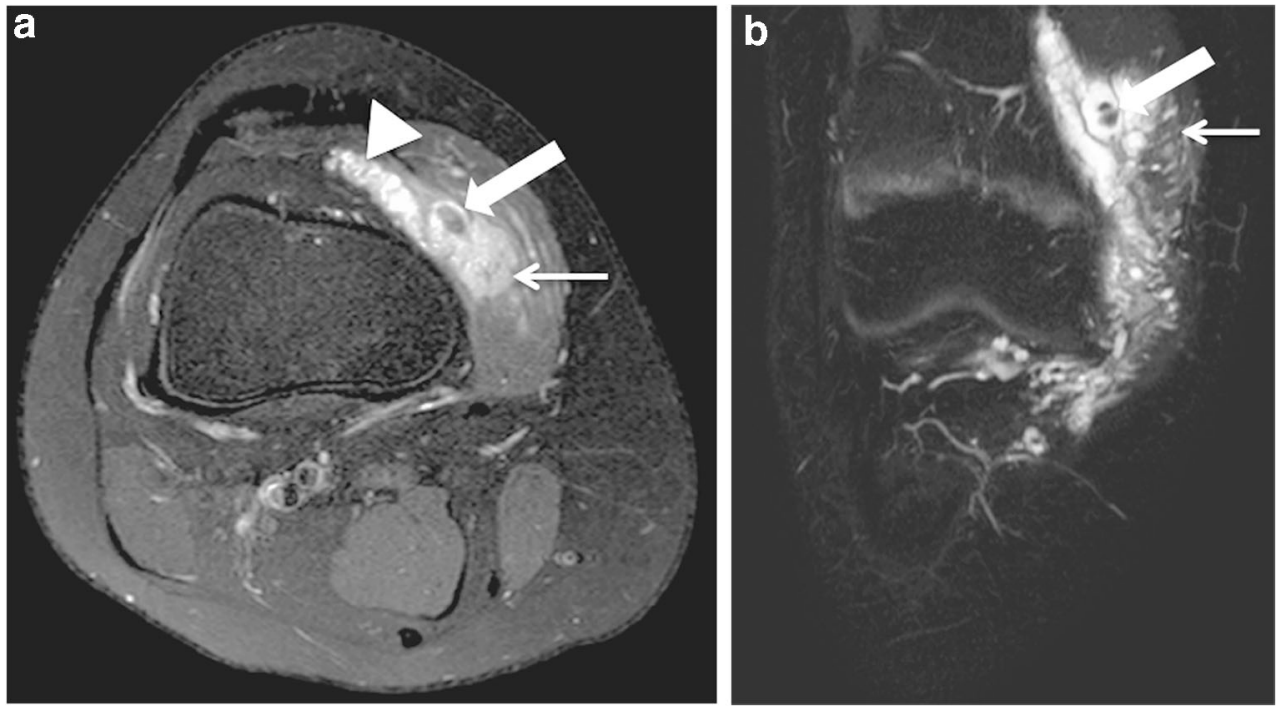

vascular spaces. Delayed (6-7 $\mathrm{min}$ ) post-contrast images are helpful because the enhancement typically increases gradually in consecutive sequences following injection (Fig. 3). Filling defects due to thrombosis may be present (Fig. 3), whereas organizing thrombi tend to enhance intensively. In suspicion of high flow vascular lesions, arteriovenous malformations and fistulas, a dynamic contrast-enhanced magnetic resonance angiography is essential to detect arteriovenous shunting presented as early filling of the draining veins. Gradient echo hemosiderin sensitive sequences, such as $\mathrm{T} 2 *$ sequences, may help detect previous bleeding in intrasynovial venous malformations. Restricted diffusion is not present in venous malformations, hence diffusion-weighted imaging is not included in our routine protocol.

In this study, the main differential diagnoses offered for venous malformations were juvenile idiopathic arthritis, pigmented villonodular synovitis and hemangioma. Although mild synovial effusions may be present in venous malformations, the synovium itself does not enhance after gadolinium as it does in inflammatory synovitis, such as rheumatoid arthritis. In pigmented villonodular synovitis, the synovial thickening has different morphology and a more heterogeneous T2 signal than in venous malformations, although hemosiderin deposits are possible in both entities.

Hemangioma is a commonly used misnomer for venous malformations in many vascular anomaly publications $[15,16]$ and was also presented as an initial diagnosis among some of our cases. Also, intra-articular venous malformations of the knee may be misleadingly designated as synovial hemangiomas in the literature [17-20]. On MRI, hemangiomas are solid lesions with uniformly high signal intensity in T2-weighted sequences, prominent feeding arteries and draining veins, and intense and homogeneous enhancement after gadolinium. These features permit ready differentiation from venous malformations on imaging. Furthermore, most hemangiomas are of the infantile type, following a course of initial growth during the first few months of life followed by spontaneous involution. In contrast, venous malformations grow commensurately with the child [7] and may not become symptomatic until later childhood. Moreover, hemangiomas are not practically encountered in the knee joint in our experience.

Only a few studies of intra-articular venous malformations have distinguished between intracapsular but extrasynovial and intrasynovial lesions $[4,5,21]$, although this is a clear determinant of the risk of chondral damage. In association with an intra-articular venous malformation, mild synovial effusion, hemosiderin deposits and thinning of cartilages are signs of recurrent bleeding and should raise a strong suspicion of an intrasynovial lesion. An important learning point is that synovial involvement may not always be evident on MRI, especially in the absence of a synovial effusion (Fig. 2). As shown by our data, it should be suspected in suprapatellar lesions with close proximity to the synovium of the suprapatellar recess and in diffuse lesions invading the intercondylar space near cruciate ligaments (Figs. 4 and 5). In our series, two patients with lesions of the suprapatellar fat pad had involvement of the suprapatellar recess not visible on MRI but detected at surgical treatment. It is important to note that the suprapatellar recess, also referred to as the suprapatellar synovial bursa, is continuous with the joint cavity and lies immediately posterior to the suprapatellar fat pad. In intra-articular venous malformations of the knee, clinicians should be made aware of this potential limitation of MRI. Diagnostic arthroscopy is one option for further investigating the synovium in unclear cases. 


\section{Conclusion}

Intra-articular venous malformations of the knee are a rare but important condition in the pediatric population. The clinical picture in children may be nonspecific and the symptoms and signs display overlap with other conditions. Although MRI usually permits the diagnosis, familiarity with vascular malformations is important for optimal imaging and accurate interpretation. Involvement of the intrasynovial cavity is a risk for hemarthrosis and progressive chondropathy but possibly underestimated by magnetic resonance images.

Acknowledgements Open access funding provided by University of Helsinki including Helsinki University Central Hospital. We would like to thank Dr Jouko Lohi for providing histopathology images for this article.

\section{Compliance with ethical standards}

\section{Conflicts of interest None}

Open Access This article is distributed under the terms of the Creative Commons Attribution 4.0 International License (http:// creativecommons.org/licenses/by/4.0/), which permits unrestricted use, distribution, and reproduction in any medium, provided you give appropriate credit to the original author(s) and the source, provide a link to the Creative Commons license, and indicate if changes were made.

\section{References}

1. Mulliken JB, Fishman SJ, Burrows PE (2000) Vascular anomalies. Curr Probl Surg 37:517-584

2. Gupta A, Kozakewich H (2011) Histopathology of vascular anomalies. Clin Plast Surg 38:31-44

3. Tsai A, Chaudry G, Spencer S et al (2011) Misdiagnosis of knee venous malformation as juvenile idiopathic arthritis. J Pediatr Orthop 31:683-690

4. Dalmonte P, Granata C, Fulcheri E et al (2012) Intra-articular venous malformations of the knee. J Pediatr Orthop 32:394-398

5. Enjolras O, Ciabrini D, Mazoyer E et al (1997) Extensive pure venous malformations in the upper or lower limb: a review of 27 cases. J Am Acad Dermatol 36:219-225
6. Bonaga S, Bardi C, Gigante C, Turra S (2003) Synovial involvement in hemangiomatosis. Arch Orthop Trauma Surg 123:102-106

7. Pireau N, Boon LM, Poilvache P, Docquier PL (2016) Surgical treatment of intra-articular knee venous malformations: when and how? J Pediatr Orthop 36:316-322

8. ISSVA Classification of Vascular Anomalies (C2018 International Society for the Study of Vascular Anomalies. Available at issva.org/ classification. Accessed 26 Aug 2019

9. Dompmartin A, Ballieux F, Thibon P et al (2009) Elevated D-dimer level in the differential diagnosis of venous malformations. Arch Dermatol 145:1239-1244

10. Laurian C, Masonni C, Wassef M et al (2018) Surgical treatment of venous malformations of the knee: treatment outcomes for 35 patients. J Pediatr Orthop 38:e325-e331

11. Hu L, Chen H, Yang X et al (2018) Joint dysfunction associated with venous malformations of the limb: which patients are at high risk? Phlebology 33:89-96

12. Hyodoh H, Hori M, Akiba $H$ et al (2005) Peripheral vascular malformations: imaging, treatment approaches, and therapeutic issues. Radiographics 25:S159-S171

13. Restrepo R (2013) Multimodality imaging of vascular anomalies. Pediatr Radiol 43:S141-S154

14. Calligaris L, Berti I, Barbi E (2015) Phleboliths as a marker of slowflow venous malformation. Arch Dis Child 100:1012-1012

15. Hassanein AH, Mulliken JB, Fishman SJ, Greene AK (2011) Evaluation of terminology for vascular anomalies in current literature. Plast Reconstr Surg 127:347-351

16. Dasgupta R, Fishman SJ (2014) ISSVA classification. Semin Pediatr Surg 23:158-161

17. Abe T, Tomatsu T, Tazaki K (2002) Synovial hemangioma of the knee in young children. J Pediatr Orthop B 11:293-297

18. Cotten A, Flipo RM, Herbaux B et al (1995) Synovial haemangioma of the knee: a frequently misdiagnosed lesion. Skeletal Radiol 24:257-261

19. Price NJ, Cundy PJ (1997) Synovial hemangioma of the knee. J Pediatr Orthop 17:74-77

20. Lopez-Oliva CL, Wang EH, Cañal JP (2015) Synovial haemangioma of the knee: an under recognised condition. Int Orthop 39:2037-2040

21. Laurian C, Franceschi C, Herbreteau D, Enjolras O (2004) Traitement chirurgical des malformations vasculaires des membres [surgical treatment of vascular malformations of lower limbs]. EMC - Chirurgie 1:100-124

Publisher's note Springer Nature remains neutral with regard to jurisdictional claims in published maps and institutional affiliations. 